\title{
Validation of the 28-day mortality prognostic performance of the modified Nutrition Risk in Critically Ill (mNUTRIC) score in a Malaysian intensive care unit
}

\author{
Wan Fadzlina Wan Muhd Shukeri ${ }^{1,2}$, Samiullah Saeed ${ }^{2}$, Azrina Md Ralib ${ }^{2}$ \& \\ Mohd Basri Mat-Nor ${ }^{2 *}$ \\ ${ }^{1}$ Department of Anaesthesiology and Intensive Care, School of Medical Sciences, \\ Universiti Sains Malaysia, Kelantan, Malaysia; ${ }^{2}$ Department of Anaesthesiology and \\ Critical Care, Kuliyyah of Medicine, International Islamic University of Malaysia, \\ Pahang, Malaysia
}

\begin{abstract}
Introduction: The mNUTRIC score is a nutritional assessment tool to identify critically ill patients with high nutritional risk who could benefit from nutritional interventions. This study was conducted to validate the 28-day mortality prognostic performance of the mNUTRIC score in a Malaysian intensive care unit (ICU). Methods: This was a retrospective cohort study of adult patients who were consecutively admitted to the ICU from January 2017 to December 2018 for $>24$ hours. Data were collected on variables required to calculate the mNUTRIC score. Patients with mNUTRIC score $\geq 5$ points were considered to be at high nutritional risk. Main outcome was 28 day mortality from all causes; ICU length of stay (LOS) and prolonged mechanical ventilation (MV) (>2 days) were secondary outcomes. Results: From a total of 432 admissions, $382(88.4 \%)$ patients fulfilled the study criteria. Seventy-seven $(20.2 \%)$ of these patients were at high nutritional risk. They had longer mean ICU LOS $(7.1 \pm 7.5$ days versus $4.2 \pm 4.0$ days, $p=0.001)$, greater proportion of prolonged MV (57.1\% versus $14.4 \%, p<0.001)$ and higher 28 -day mortality $(44.2 \%$ versus $10.2 \%$, $p<0.001)$ compared to patients with low mNUTRIC score ( $\leq 4$ points). High mNUTRIC score predicted 28-day mortality with area under the curve (AUC) of 0.797 (95\% confidence interval: 0.738-0.856). Conclusion: High mNUTRIC score was associated with a higher 28-day mortality. The prognostic performance for 28-day mortality of the mNUTRIC score is clinically valid as indicated by AUC $>0.7$ and is comparable to the results of other validation studies. In addition, patients with high mNUTRIC score had increased ICU LOS and prolonged MV.
\end{abstract}

Keywords: Nutritional status, critically ill, mortality

\section{INTRODUCTION}

It is the standard practice to provide nutritional support to critically ill patients in order to treat existing malnutrition and minimise adverse clinical outcomes. Adequate nutritional support for these patients has been shown to improve clinical outcomes, particularly in patients at 'high nutritional risk' (Kondrup et al., 2003). Therefore, identifying critically ill patients who are at high risk of malnutrition is an important role of

\footnotetext{
*Corresponding author: Associate Prof. Dr. Mohd Basri Mat-Nor Department of Anaesthesiology and Critical Care, Kuliyyah of Medicine, International Islamic University of Malaysia, Pahang, Malaysia Tel: +609-5912610; E-mail: basri.matnor@gmail.com doi: https://doi.org/10.31246/mjn-2019-0074
} 
intensivists. Nonetheless, evaluating nutritional risk of critically ill patients remains a challenge for intensivists for a variety of reasons. First, critically ill patients are often intubated which poses a communication barrier to obtaining an accurate dietary history. Second, anthropometric measures may be obscured by oedema while voluntary handgrip strength is impractical in unconscious patients. Third, laboratory measures, such as pre-albumin and albumin levels, lymphocyte counts and transferrin, are abnormal in critical illness (Bersten \& Soni, 2014).

In 2011, Heyland et al. introduced the Nutrition Risk in Critically Ill (NUTRIC) score, the first nutritional risk assessment tool that was developed for intensive care unit (ICU) patients (Heyland et al., 2011). The score identifies high nutritional risk patients who will benefit from aggressive nutritional support by linking starvation, inflammation and outcomes (Heyland et al., 2011). It consists of six variables, which are age, the Acute Physiology and Chronic Health Evaluation (APACHE) II score, the Sequential Organ Failure Assessment (SOFA) score, the number of co-morbidities, the number of days from hospital to ICU admission, and blood interleukin-6 (IL-6) concentration. Patients get 1-3 points for each variable and the highest score adds up to 10 points. Those who score $\geq 5$ points are considered to be at high risk for malnutrition.

However, the IL-6 levels are not commonly measured in many institutions. Heyland et al. (2011) have stated that IL- 6 only increased the area under the curve (AUC) by 0.007 (from 0.776 to 0.783 ), being neither clinically nor statistically different. They have therefore suggested that in settings where IL-6 is not available this parameter could be dropped from the calculation of the score. This adjusted score is called the modified NUTRIC (mNUTRIC) score (Table 1). Because the IL- 6 component is dropped, the mNUTRIC score adds up to 9 points. Rahman et al. (2016) have demonstrated the validity of the mNUTRIC Score in an external population and this was followed by several other validation studies (De Vries et al., 2018; Jeong et al., 2018; Jung et al., 2018; Mendes et al., 2017; Moretti et al., 2014). Nonetheless, these studies were mainly conducted in the setting of western ICUs or in high-income countries. The evidence for validation of the mNUTRIC score in the low- and middle-income countries are very limited (Kalaiselvan, Renuka \& Arunkumar, 2017). Thus, the purpose of the present study was to validate the prognostic performance of the mNUTRIC score in a Malaysian ICU, as reflected by the impact on 28-day mortality, length of stay (LOS) in the ICU and duration of mechanical ventilation (MV).

\section{MATERIALS AND METHODS}

This was a retrospective cohort study that was conducted in the ICU of International Islamic University (IIUM) Medical Centre in Pahang, Malaysia from 12 January 2017 to 31 December 2018. The study was approved by the IIUM research and ethics committee (IREC). Written informed consent of the patients could not be obtained because of the retrospective nature of the study design. Consecutive adult patients (aged $\geq 18$ years) admitted to the ICU for $>24$ hours were included in the study. Those who were readmitted and with ICU LOS of $<24$ hours were excluded from the analysis. We reviewed the ICU charts and the electronic medical records to collect data on baseline demographic and clinical characteristics, the parameters required to calculate the mNUTRIC score and outcome data. The main outcome was 28-day mortality from all causes; 
Table 1. The parameters required for the calculation of the mNUTRIC score

\begin{tabular}{lcc}
\hline Variable $^{\dagger}$ & Range & Points \\
\hline Age (years) & $<50$ & 0 \\
& 50 to $<75$ & 1 \\
& $\geq 75$ & 2 \\
APACHE II & $<15$ & 0 \\
& 15 to $<20$ & 1 \\
& 20 to 28 & 2 \\
SOFA & $\geq 28$ & 3 \\
& $<6$ & 0 \\
Number of co-morbidities & 6 to $<10$ & 1 \\
& $\geq 10$ & 2 \\
Days from hospital to ICU admission & 0 to 1 & 0 \\
& $\geq 2$ & 1 \\
& 0 to $<1$ & 0 \\
Sum of points & $\geq 1$ & 1 \\
\hline 5-9 & Category & Explanation \\
\hline 0-4 & High score & Associated with worse clinical \\
& & outcomes (mortality, ventilation) \\
\hline APACHE, Acute & The patients have a low \\
& & malnutrition risk \\
\hline
\end{tabular}

${ }_{\dagger}^{\dagger}$ APACHE, Acute Physiology and Chronic Health Evaluation; ICU, intensive care unit; SOFA, Sequential Organ Failure Assessment

ICU LOS and prolonged MV (>2 days) were secondary outcomes.

All statistical analyses were performed using SPSS version 24.0 (IBM, Armonk, New York, USA) and MedCalc for Windows, version 17.5.5 (MedCalc Software, Ostend, Belgium). Continuous variables were presented as mean \pm standard deviation (SD) while categorical variables were presented as frequencies (percentages). Patients with a mNUTRIC score $\geq 5$ points were considered to be at high nutritional risk while those with score $\leq 4$ points were considered low nutritional risk. Univariate comparison of the continuous variables between these two groups was analysed using the independent t-test. Univariate comparison of the categorical variables between these two groups was analysed using the chi-squared test. $P<0.05$ was considered statistically significant for all tests. The prognostic performance of the mNUTRIC score was assessed by the AUC of the receiver operating characteristic (ROC) curve of sensitivity against (100-specificity) across a series of the score's readings. The AUC ranges from 0.5 (no discrimination) to 1.0 (perfect discrimination). Clinical validity was assumed to be at an AUC of $>0.7$ (Bewick, Cheek \& Ball, 2004). The sensitivity, specificity, positive predictive value (PPV), negative predictive value (NPV), positive likelihood ratio (PLR) and negative likelihood ratio (NLR) of the score were determined from the ROC curve analysis. The AUC, sensitivity, 
specificity, predictive values and likelihood ratios were reported with 95\% confidence interval (CI).

\section{RESULTS}

\section{Baseline demographic and clinical characteristics}

A total of 432 patients were admitted to the ICU during the study period. Fifty $(11.6 \%)$ patients were excluded from the study; $19(4.4 \%)$ patients were underaged, 23 (5.3\%) patients were readmission cases and $8(1.9 \%)$ patients had an ICU LOS of $<24$ hours (Figure 1). Thus, the data of 382 (88.4\%) patients were analysed. A total of 77 of the 382 $(20.2 \%)$ patients were at high nutritional risk (mNUTRIC score $\geq 5$ points). The baseline demographic and clinical characteristics of the patients who were included in the analyses are shown in Table 2. The patients who were at high nutritional risk were older (66 49 years versus $54 \pm 16$ years, $p<0.001$ ) and the majority of them were medical cases $(66.2 \%$ versus $44.9 \%, p=0.001)$. Sepsis $(26.0 \%$ versus $14.8 \%, p=0.019)$ and shock $(26.0 \%$ versus $11.5 \%, p=0.001)$ were more commonly noted as the reasons for ICU admission among the high nutritional risk patients compared to the patients with low nutritional risk. The mean mNUTRIC score in the overall population was $2.9 \pm 1.9$ points. As expected, the mean score was higher in the high nutritional risk than in the low nutritional risk group $(5.8 \pm 0.9$ points versus $2.2 \pm 1.4$ points, $p<0.001$ ).

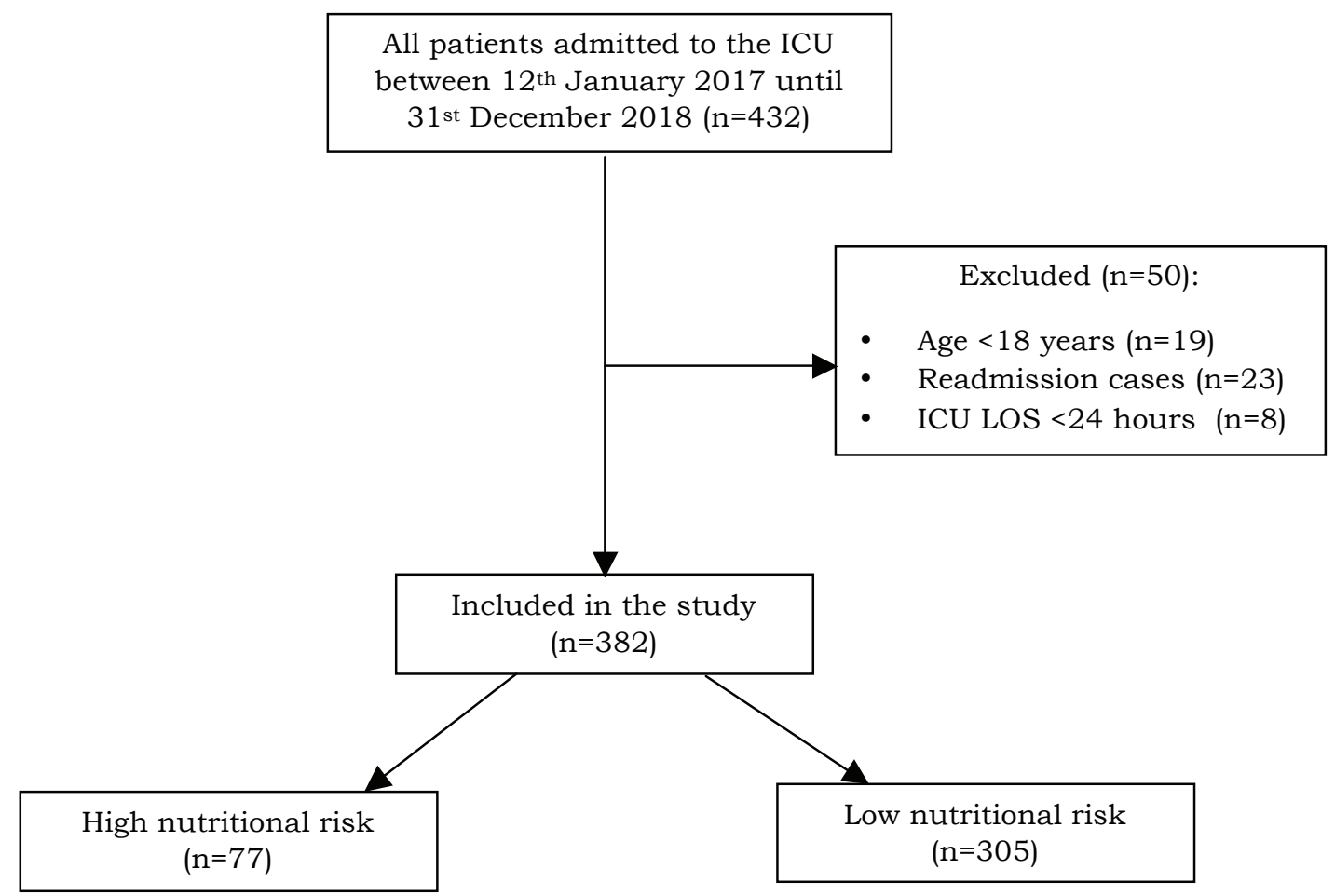

Figure 1. Flowchart of patients' selection

Note: ICU, Intensive care unit; LOS, length of stay 
Table 2. Baseline demographics and clinical characteristics

\begin{tabular}{|c|c|c|c|c|}
\hline Variables $^{\dagger}$ & $\begin{array}{l}\text { All patients } \\
\quad(N=382)\end{array}$ & $\begin{array}{c}\text { Low nutritional } \\
\text { risk } \\
(n=305)\end{array}$ & $\begin{array}{c}\text { High nutritional } \\
\text { risk } \\
(n=77)\end{array}$ & $p$ \\
\hline \multicolumn{5}{|l|}{ Demographics } \\
\hline Age (years), mean $\pm S D$ & $57 \pm 16$ & $54 \pm 16$ & $66 \pm 9$ & $<0.001$ \\
\hline \multicolumn{5}{|l|}{ Sex } \\
\hline Male, n (\%) & $216(56.5)$ & $168(55.1)$ & $48(62.3)$ & 0.251 \\
\hline Female, n (\%) & $166(43.5)$ & 137 (44.9) & $29(37.7)$ & \\
\hline BMI $\left(\mathrm{kg} / \mathrm{m}^{2}\right)$, mean $\pm S D$ & $26.3 \pm 7.5$ & $26.0 \pm 5.4$ & $27.6 \pm 14.1$ & 0.811 \\
\hline \multicolumn{5}{|l|}{ Clinical } \\
\hline \multicolumn{5}{|l|}{ Admission category, n (\%) } \\
\hline Medical & $188(49.2)$ & $137(44.9)$ & $51(66.2)$ & 0.001 \\
\hline Surgical & $194(50.8)$ & $168(55.1)$ & $26(33.8)$ & 0.001 \\
\hline \multicolumn{5}{|c|}{ Reasons for ICU admission, n(\%) } \\
\hline Post-operative care & $127(33.2)$ & $120(39.3)$ & $7(9.1)$ & $<0.001$ \\
\hline Respiratory failure & $107(28)$ & $83(27.2)$ & $24(31.2)$ & 0.490 \\
\hline Sepsis & $65(17)$ & $45(14.8)$ & $20(26.0)$ & 0.019 \\
\hline Shock & $55(14.4)$ & 35 (11.5) & $20(26.0)$ & 0.001 \\
\hline Neurological deterioration & $27(7)$ & $21(6.9)$ & $6(7.8)$ & 0.781 \\
\hline Toxicity & $1(0.4)$ & $1(0.3)$ & $0(0)$ & 0.615 \\
\hline \multicolumn{5}{|l|}{ Severity of illness, mean $\pm S D$} \\
\hline APACHE II & $13.0 \pm 7.7$ & $10.8 \pm 6.1$ & $23.1 \pm 5.7$ & $<0.001$ \\
\hline SOFA & $4.0 \pm 3.8$ & $2.9 \pm 2.9$ & $8.4 \pm 3.6$ & $<0.001$ \\
\hline mNUTRIC score & $2.9 \pm 1.9$ & $2.2 \pm 1.4$ & $5.8 \pm 0.9$ & $<0.001$ \\
\hline
\end{tabular}

${ }^{\dagger}$ APACHE, Acute Physiologic Assessment and Chronic Health Evaluation; BMI, body mass index; ICU, Intensive care unit; mNUTRIC, modified Nutritional Risk in Critically Ill; SOFA, Sequential Organ Failure Assessment

\section{Prognostic performance of the mNUTRIC score}

The outcomes of the overall population and as stratified by the nutritional risk are presented in Table 3 . The primary outcome of 28-day mortality was reached in $65(17.0 \%)$ patients. Patients who were at high nutritional risk had higher 28-day mortality compared to patients at low (mNUTRIC score $\leq 4$ points) nutritional risk $(44.2 \%$ versus $10.2 \%, p<0.001)$. The mNUTRIC score on a full scale (0-9) predicted mortality with AUC of $0.797 \quad(95 \%$ CI, $0.738-$ $0.856)$, indicating a very good prognostic performance of the score in our cohort (Figure 2).
At the optimal cut-off of 6 points, the mNUTRIC score showed the following characteristics:

Sensitivity: $18.5 \%$ (95\% CI, 9.9-30.0\%)

Specificity : 98.4\% (95\% CI, 96.4-99.5\%), PPV : $\quad 70.6 \%(95 \%$ CI, 46.7-86.8\%), NPV : $\quad 85.5 \%(95 \%$ CI, 84.0-86.9\%), PLR : $\quad 11.7(95 \% \mathrm{CI}, 4.3-32.1 \%)$

NLR : $\quad 0.8(95 \%$ CI, $0.7-0.9 \%)$

In addition, patients who were at high nutritional risk also had longer mean ICU LOS (7.1 \pm 7.5 days versus 4.2 \pm 4.0 days, $p=0.001$ ) and greater proportion of prolonged MV (57.1\% versus $14.4 \%$, $p<0.001)$. 
Table 3. Comparison of outcomes of patients with high nutritional risk (mNUTRIC score $\geq 5$ points) and low nutritional risk (mNUTRIC score $\leq 4$ points)

\begin{tabular}{|c|c|c|c|c|}
\hline Variables $^{\dagger}$ & $\begin{array}{l}\text { All patients } \\
(N=382)\end{array}$ & $\begin{array}{c}\text { Low nutritional } \\
\text { risk } \\
(n=305)\end{array}$ & $\begin{array}{c}\text { High nutritional } \\
\text { risk } \\
(n=77)\end{array}$ & $p^{\ddagger}$ \\
\hline ICU LOS (days), mean $\pm S D$ & $4.8 \pm 5.1$ & $4.2 \pm 4.0$ & $7.2 \pm 7.5$ & 0.001 \\
\hline $\begin{array}{l}\text { Prolonged mechanical } \\
\text { ventilation ( }>2 \text { days), } \mathrm{n}(\%)\end{array}$ & $88(23)$ & $44(14.4)$ & $44(57.1)$ & $<0.001$ \\
\hline 28-day mortality, n (\%) & 65 (17) & $31(10.2)$ & $34(44.2)$ & $<0.001$ \\
\hline
\end{tabular}

${ }^{\dagger} \mathrm{ICU}$, intensive care unit; LOS, length of stay

₹The results of the comparison between the two groups was analysed by the independent $\mathrm{t}$-test for continuous variable or the chi-squared test for categorical variables

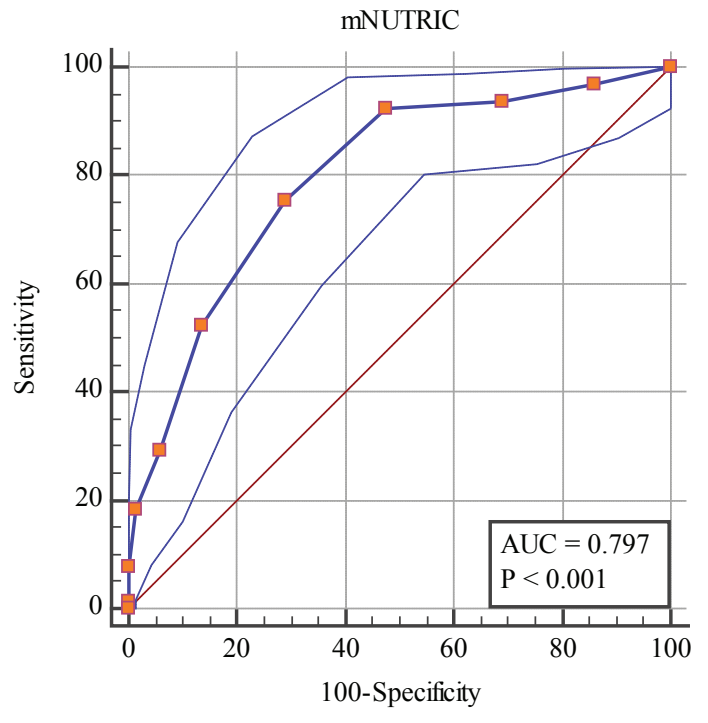

Figure 2. Prognostic performance of the mNUTRIC score on a scale of 0-9 in predicting 28-day mortality in critically ill patients

Note: mNUTRIC, modified Nutrition Risk in Critically Ill; AUC, area under the curve

\section{DISCUSSION}

As identification of critically ill patients with high nutritional risk is important to reduce poor clinical outcomes, the need for an easy-to-implement, low cost, highly-effective scoring system is undeniable. Many of the traditionally used nutritional screening tools such as the Malnutrition Universal Screening Tool, the Nutritional Risk Screening (NRS 2002) and the Subjective Global Assessment use anthropometric measurements and the history of dietary intake or weight loss to identify patients at nutritional risk (Detsky et al., 1987; Elia, 2003; Kondrup et al., 2003). Anthropometric measures can be unreliable in ICU patients because of underlying oedema and a reliable history of dietary intake or weight loss is difficult to obtain as these patients are often intubated and sedated. The NUTRIC score was the first nutritional risk assessment tool that had been developed specifically for ICU patients. Although the NUTRIC score is effective, the inclusion of the costly and often unavailable IL-6 measurement makes it unattractive for widespread implementation. As such, the mNUTRIC score appears to be the more promising nutritional risk assessment tool but further validation is warranted.

The main objective of this study was to validate the prognostic performance of the mNUTRIC score mainly for mortality, in our local ICU setting. In this study, we found that $20.2 \%$ of patients admitted to our ICU were at high nutritional risk (mNUTRIC score $\geq 5$ points). This percentage was lower than that reported 
by Mendes et al. (2017) (48.6\%) and Kalaiselvan et al. (2017) (42.5\%) who also employed the mNUTRIC score in assessing the prevalence of malnutrition in ICU patients. In our cohort, the mNUTRIC score had a good performance in predicting mortality, as indicated by an AUC of 0.797. This is comparable to two other recent validation studies where the AUC of the mNUTRIC score for 28-day mortality was 0.768 in a Dutch ICU (De Vries et al., 2018) and 0.757 in a Korean ICU (Jeong et al., 2018). This finding suggests that the mNUTRIC score is a good prognostic substitute for the NUTRIC in assessing nutritional risk in the ICU. As with the Korean study by Jeong et al. (2018), we found that the cut-off of 6 points for the mNUTRIC score was better at predicting 28-day mortality than the cut-off of 5 points as suggested by the original NUTRIC score. Our study found that the mNUTRIC score was also valid in predicting other clinical outcomes such as longer ICU LOS and prolonged $\mathrm{MV}$, in line with the previous findings by others (De Vries et al., 2018; Kalaiselvan et al., 2017; Mendes et al., 2017).

This study has several strengths. To our knowledge, our study is the first to validate the prognostic utility of the mNUTRIC score in the local ICU setting. Nutritional risk assessment is an important part of the management of critically ill patients but is often overlooked. Unlike the original NUTRIC score, with the exception of IL-6 level, the mNUTRIC score is easier to calculate. Given our results, we recommend the introduction of the mNUTRIC score for the nutritional risk assessment of our critically ill patients considering its suitability and feasibility. The mNUTRIC score may also have utility in the design and interpretation of clinical trials of nutrition in the ICU setting. Second, our study strengthens the evidence obtained by a previous study (Jeong et al., 2018) that the optimal cut-off of the mNUTRIC score for mortality was 6 points, in contrast to the cut-off of 5 points as suggested by the original NUTRIC score. Third, our study was conducted in a cohort of mixed medical and surgical ICU patients; this may allow the general application of the results obtained to all critically ill patients.

Nevertheless, our study has several limitations. First, we did not calculate the nutritional support provided to the patients. Therefore, the association between nutritional adequacy, mNUTRIC score and mortality could not be confirmed by our results. However, this was not the main aim of the study. This study was conducted primarily with the aim of validating the prognostic performance of the mNUTRIC score in our local ICU setting. The second limitation of our study was our inability to obtain IL-6 levels of the patients. Even though our intent was to examine the score without IL-6, a comparison with IL-6 would have allowed us to see the difference between the NUTRIC and mNUTRIC scores. Third, it may also be a limitation to the current validation of the mNUTRIC score that it is based on 28-day mortality, which was the only mortality data available for use in this dataset. Choosing longer term outcomes, such as 90-day mortality or some measure of functional status at hospital discharge may have yielded different but important results. Fourth is the limitation related to that of the NUTRIC score itself; clinicians may argue that there is little need for another risk score since those such as APACHE II or SOFA score are available. The calculation of the score can be cumbersome, thus limiting its clinical utility. Others correctly point out that the NUTRIC score does not contain traditional nutrition variables. Unfortunately, in an ICU setting, these variables depend on history from family members, which can be inconsistent. 
Finally, our study was conducted at a single centre, and its findings cannot be generalised to the wider external population.

\section{CONCLUSION}

The ability of the mNUTRIC score to discriminate between high and low risk for 28-day mortality in Malaysian ICU patients is clinically valid and comparable with that found in previous validation studies. Our results also confirmed the association of high mNUTRIC scores with prolonged MV and longer ICU stay. We therefore suggest the introduction of the mNUTRIC score for the nutritional risk assessment of critically ill patients in Malaysian ICUs. However, further multicentre prospective studies are warranted to show the effect of nutritional interventions in critically ill patients.

\section{Acknowledgement}

This work was supported by the International Islamic University Malaysia Research Initiative Grant RIGS 16-113-0277.

\section{Authors' contributions}

WFWMS conducted the study, data analysis and interpretation, assisted in drafting of the manuscript, reviewed the manuscript; SS undertook data collection and reviewed the manuscript; AMR advised on the data analysis and interpretation and reviewed the manuscript; MBMN, the principal investigator, conceptualised and designed the study, prepared the draft manuscript and reviewed the final manuscript.

\section{Conflict of interest}

The authors declare no conflict of interest.

\section{List of abbreviations used in the text}

APACHE, Acute Physiology and Chronic Health Evaluation; ICU, intensive care unit; IL-6, interleukin-6; LOS, length of stay; mNUTRIC, modified Nutrition Risk in Critically Ill; NUTRIC, Nutrition Risk in Critically Ill; MV, mechanical ventilation; SOFA, Sequential Organ Failure Assessment

\section{References}

Bersten AD \& Soni N (2014). Oh's Intensive Care Manual (Seventh Ed). Butterworth-Heinemann Elsevier, London, United Kingdom.

Bewick V, Cheek L \& Ball J (2004). Statistics review 13: receiver operating characteristic curves. Crit Care 8(6):508-512. https://doi. org/10.1186/cc3000

De Vries MC, Koekkoek W, Opdam MH, Van Blokland D \& Van Zanten AR (2018). Nutritional assessment of critically ill patients: validation of the modified NUTRIC score. Eur $J$ Clin Nutr 72:428-435. https://doi.org/10.1038/ s41430-017-0008-7

Detsky AS, McLaughlin JR, Baker JP, Johnston N, Whittaker S, Mendelson RA \& Jeejeebhoy KN (1987). What is subjective global assessment of nutritional status? J Parenter Enteral Nutr 11(1):8-13.

Elia M (2003). The 'MUST' report. Nutritional screening for adults: a multidisciplinary responsibility. Development and use of the 'Malnutrition Universal Screening Tool' (MUST) for adults. British Association for Parenteral and Enteral Nutrition (BAPEN), Redditch.

Heyland DK, Dhaliwal R, Jiang X \& Day AG (2011). Identifying critically ill patients who benefit the most from nutrition therapy: the development and initial validation of a novel risk assessment tool. Crit Care 15:R268. https://doi.org/10.1186/cc10546

Jeong DH, Hong SB, Lim CM, Koh Y, Seo J, Kim Y, Min JY \& Huh JW (2018). Comparison of accuracy of NUTRIC and modified NUTRIC scores in predicting 28-day mortality in patients with sepsis: a single center retrospective study. Nutrients 10(7):911. https://doi.org/10.3390/ nu10070911

Jung YT, Park JY, Jeon J, Kim MJ, Lee SH \& Lee JG (2018). association of inadequate caloric supplementation with 30-day mortality in critically ill postoperative patients with high modified NUTRIC Score. Nutrients 10(11):1589. https://doi.org/10.3390/nu10111589

Kalaiselvan M, Renuka MK \& Arunkumar AS (2017). Use of nutrition risk in critically ill (NUTRIC) score to assess nutritional risk in mechanically ventilated patients: a prospective observational study. Indian $J$ Crit Care Med 21(5):253-256. ijccm.ijccm_24_17 https://doi.org/10.4103/ 
Kondrup J, Ramussen HH, Hamberg O, Stanga $Z$ \& Ad Hoc ESPEN Working Group (2003). Nutritional risk screening (NRS 2002): a new method based on an analysis of controlled clinical trials. Clin Nutr 22(3):321-336. https: / / doi.org/10.1016/S0261-5614(02)00214-5

Mendes R, Policarpo S, Fortuna P, Alves M, Virella D $\&$ Heyland DK \& the Portuguese NUTRIC Study Group (2017). Nutritional risk assessment and cultural validation of the modified NUTRIC score in critically ill patients-a multicenter prospective cohort study. J Crit Care 37:45-49. https://doi.org/10.1016/j.jcrc.2016.08.001
Moretti D, Bagilet DH, Buncuga M, Settecase CJ, Quaglino MB \& Quintana R (2014). Study of two variants of nutritional risk score "NUTRIC" in ventilated critical patients. Nutr Hosp 29(1):166-172. nh.2014.29.1.7001

Rahman A, Hasan RM, Agarwala R, Martin C, Day AG \& Heyland DK (2016). Identifying critically-ill patients who will benefit most from nutritional therapy: further validation of the "modified NUTRIC" nutritional risk assessment tool. Clin Nutr 35(1):158-162. https://doi. org/10.1016/j.clnu.2015.01.015 